\title{
Fuzzy Active Disturbance Rejection Attitude Control of Spacecraft with Unknown Disturbance and Parametric Uncertainty
}

\author{
Chenxing Zhong, Yu Guo* and Lu Wang \\ School of Automation, Nanjing University of Science and Technology \\ Nanjing, 210094, China \\ *corresponding author, e-mail: guoyu@njust.edu.cn
}

\begin{abstract}
Based on active disturbance rejection control (ADRC) and fuzzy logic control, a control scheme for attitude maneuver control of spacecraft is proposed. In which a maneuver path is planned to reduce overshoot and improve rapidity, and an extended state observer is designed to cope with unknown disturbances and parameter uncertainties. Furthermore, in order to improve dynamic performance of the control system, the gains of the nonlinear feedback control law are adjusted by fuzzy control online. Simulation results have convincingly demonstrated the effectiveness and robustness of the proposed control scheme.
\end{abstract}

Keywords: Spacecraft attitude control, ADRC, Fuzzy logic control, Disturbance, Parametric uncertainty.

\section{Introduction}

With the development of space technology, the missions of spacecraft become much more complicated, which highly demand the maneuver rapidity and pointing accuracy for the spacecraft attitude control system. Whereas, from practical point of view, an orbiting spacecraft, as well as its attitude control system, is inevitably affected by various external disturbance torques, such as radiation torque, gravitational torque and so forth. In addition, the dynamics of a spacecraft is of nonlinear and its parameters are time-varying on-orbit operation. As a result, the parameters are always uncertain in spacecraft. These issues pose a great challenge to achieve both of rapid maneuver and high-precision pointing for an attitude control system of a spacecraft.

In recent years, the attitude control problem of spacecraft has attracted a great deal of attention. One of the major problems encountered in practice for the design of attitude controller is how to compensate for the external disturbances and parametric uncertainties. Various new control methods have been proposed to address the issue, such as backstepping control, adaptive control, sliding mode control and so forth [1-6]. Chen [2] proposed an adaptive control approach to deal with the attitude control and disturbance rejection problem of rigid spacecraft for a class of persistent disturbances with unknown phase angles and amplitudes but known frequencies. In Ref [3], a robust backstepping control approach using an arctangent nonlinear tracking function was proposed to cope with parametric uncertainties for the spacecraft slew maneuver. Xia [4] proposed an attitude controller combing adaptive sliding mode control with an extended state observer, in which known bounded external disturbances and parametric uncertainties in inertia matrix can be compensated. Although those control methods have been shown effectiveness, their control performances are heavily related with the upper bound information about the parametric uncertainty which is hard to be obtained in advance in practical attitude control systems. Moreover, it should be 
pointed out that most of the previous control methods also require to known some prior information about the disturbances, such as upper bound, frequency and so forth. As a matter of fact, the external disturbances are unknown in space and their prior information is also difficult to be obtained precisely.

For the defects described above in those control methods, active disturbance rejection control(ADRC), which does not require any prior information and is good at rejecting disturbances and parametric uncertainties [7,8], provide a possible effective way to achieve good performance for a spacecraft attitude control. Lai [9] designed an active disturbance rejection controller for spacecraft attitude maneuver to gain high precision and high stability. Based on ADRC and path planning, Zhong [10] proposed a control strategy for spacecraft without angular velocity sensors, in which the angular velocity can be estimated with an extended state observer and the external disturbance can be compensated effectively. However, it is not easy to set appropriate parameters of the nonlinear feedback law in the ADRC controller to achieve ideal dynamic performance of the control system. Aiming to solve the issue, in this paper we introduce fuzzy logic control, which has the abilities of adaptive reasoning and parameter estimation, to adjust the parameters of nonlinear feedback law of ADRC. With the advantages of the above ADRC and fuzzy logic control, we propose a novel fuzzy active disturbance rejection control scheme for a rigid spacecraft with unknown disturbances and parametric uncertainties. The main contributions of this paper lie in the following:

(1) Via planning maneuver path for transient process, the attitude maneuver rapidity can be improved and overshoot can be reduced.

(2) Being different from most of the existing control algorithms, the proposed controller does not require any prior information of external disturbances and parametric uncertainties;

(3) Fuzzy logic control is introduced in ADRC attitude controller to adjust the parameters of nonlinear feedback law online, which improve the dynamic performance greatly.

The rest of this paper is organized as follows. In the next section, the spacecraft kinematics and dynamics are given. The design of transient process and fuzzy ADRC controller is presented in the section 3 and 4 , respectively. Then, the simulation is carried out to demonstrate the effectiveness of the proposed control scheme, followed by the conclusion.

\section{Spacecraft Model Description}

The mathematical model of a rigid spacecraft is described by two sets of dynamic and kinematic equations.

The dynamic equation of a rigid spacecraft is governed by the following nonlinear equation [11]:

$$
J \dot{\omega}+\omega^{\times} J \omega=u+d
$$

where $\boldsymbol{J} \in \boldsymbol{R}^{3 \times 3}$ is the inertia matrix of the spacecraft which is symmetric and positive-definite, $\boldsymbol{\omega} \in \boldsymbol{R}^{3 \times 1}$ is the angular velocity of the body frame relative to a reference frame, $\boldsymbol{u} \in \boldsymbol{R}^{3 \times 1}$ is control torque, $\boldsymbol{d} \in \boldsymbol{R}^{3 \times 1}$ is the external disturbance torques including constant disturbance torques and periodic disturbance torques. $\boldsymbol{\omega}^{\times}$is the skew symmetric matrix of $\boldsymbol{\omega}$, that is,

$$
\boldsymbol{\omega}^{\times}=\left[\begin{array}{ccc}
0 & -\omega_{3} & \omega_{2} \\
\omega_{3} & 0 & -\omega_{1} \\
-\omega_{2} & \omega_{1} & 0
\end{array}\right] .
$$


Considering the parametric uncertainty in inertia matrix of the spacecraft, we denote the inertial matrix as:

$$
\boldsymbol{J}=\boldsymbol{J}_{0}+\boldsymbol{J}_{\Delta},
$$

where $J_{0}$ stands for the nominal part of the inertia matrix, $J_{\Delta}$ stands for its uncertainty. Then, the dynamic equation can be written as:

$$
J_{0} \dot{\omega}=-\omega^{\times} J_{0} \omega-\omega^{\times} J_{\Delta} \omega+u-J_{\Delta} \dot{\omega}+d
$$

To avoid singularity of Euler angles, we describe the attitude kinematic equation of a spacecraft in terms of unit quaternion as follows [11]:

$$
\dot{\boldsymbol{q}}=\frac{1}{2}\left[q_{4} \mathbf{E}+\boldsymbol{q}_{\mathrm{v}}^{\times} ;-\boldsymbol{q}_{\mathrm{v}}^{\mathrm{T}}\right] \boldsymbol{\omega},
$$

where $\boldsymbol{q}=\left[\boldsymbol{q}_{\mathrm{v}}, q_{4}\right]=\left[\begin{array}{llll}q_{1} & q_{2} & q_{3} & q_{4}\end{array}\right]^{\mathrm{T}}$, subjects to the unity length constraint, i.e. $\boldsymbol{q}^{\mathrm{T}} \boldsymbol{q}=1$. Obviously, $\boldsymbol{q}_{\mathrm{v}}=\left[\begin{array}{lll}q_{1} & q_{2} & q_{3}\end{array}\right]^{\top} . \mathbf{E} \in \mathrm{R}^{3 \times 3}$ is an identity matrix herein.

The quaternion error $\boldsymbol{q}_{\mathrm{e}}$ and angular velocity error $\omega_{\mathrm{e}}$ are defined as follows [11]:

$$
\begin{aligned}
& \boldsymbol{q}_{\mathrm{e}}=\boldsymbol{q}_{\mathrm{d}}^{-1} \otimes \boldsymbol{q} \\
& \boldsymbol{\omega}_{\mathrm{e}}=\boldsymbol{\omega}-\boldsymbol{\omega}_{\mathrm{r}},
\end{aligned}
$$

where $\boldsymbol{q}_{\mathrm{d}}^{-1}$ is the inverse of the desired quaternion, the quaternion inverse is defined as

$$
\boldsymbol{q}^{-1}=\left[-q_{1},-q_{2},-q_{3}, q_{4}\right]^{\mathrm{T}} \quad \otimes \quad \otimes \quad \text { represents } \quad \text { quaternion } \quad \text { multiplication, }
$$

$\boldsymbol{q}_{e}=\left[\boldsymbol{q}_{\mathrm{ev}}, q_{4}\right]=\left[\begin{array}{llll}q_{e 1} & q_{e 2} & q_{e 3} & q_{e 4}\end{array}\right]^{\mathrm{T}}, \boldsymbol{q}_{\mathrm{ev}}=\left[\begin{array}{lll}q_{e 1} & q_{e 2} & q_{e 3}\end{array}\right]^{\top}, \boldsymbol{\omega}_{\mathrm{r}}=\boldsymbol{R}\left(\boldsymbol{q}_{\mathrm{e}}\right) \boldsymbol{\omega}_{\mathrm{d}}$. In (6), we also have $\dot{\boldsymbol{\omega}}_{\mathrm{r}}=\boldsymbol{R}\left(\boldsymbol{q}_{\mathrm{e}}\right) \dot{\boldsymbol{\omega}}_{\mathrm{d}}-\boldsymbol{\omega}_{\mathrm{e}}^{\times} \boldsymbol{R}\left(\boldsymbol{q}_{\mathrm{e}}\right) \boldsymbol{\omega}_{\mathrm{d}}$, where $\boldsymbol{\omega}_{\mathrm{d}}$ is the desired angular velocity, and $\boldsymbol{R}\left(\boldsymbol{q}_{\mathrm{e}}\right)$ is the transformation matrix from the orbit coordinate frame to the body coordinate frame.

\section{Expected Transient Process Design}

During the initial period of attitude maneuver, the maneuver command signal in the form of step may lead to large overshoot [9]. Aiming at achieving high stability and rapidity of attitude maneuver, we design an expected transient process by planning the expected angular velocity signal of maneuver path as an isosceles triangle curve. We define the slope of isosceles triangle angular velocity curve according to the permitted maximum angular acceleration, which can improve maneuver rapidity. Then, the corresponding expected attitude quaternion $\boldsymbol{q}_{\mathrm{d}}$ can be obtained by taking integral of expected angular velocity. In this way, a smooth and continuous maneuver path can be obtained. For a single-axis, we plan the maneuver path as follows:

$$
\omega_{\mathrm{d}}= \begin{cases}a t, & 0 \leq t \leq T_{1} \\ a T_{1}-a\left(t-T_{1}\right), & T_{1}<t \leq 2 T_{1} \\ 0, & t>2 T_{1}\end{cases}
$$

where $\omega_{d}$ stands for the planned angular velocity, $\boldsymbol{a}$ is a constant which stands for angular acceleration, $t$ stands for time, $T_{1}=\sqrt{\left|\theta_{\mathrm{d}}-\theta_{0}\right| / a}$ is the duration of uniform 
acceleration and deceleration, where $\theta_{0}$ and $\theta_{\mathrm{d}}$ stand for the initial attitude angle and desired attitude angle, respectively.

The transient processes for the other two axes are similar.

\section{Design of Fuzzy ADRC Controller}

From equations (3) and (4), it can be obviously seen that the attitude dynamics and kinematic equations of a spacecraft are of strongly nonlinear and multiple input multiple output. There also exist external disturbances and the parametric uncertainty in inertia matrix. In order to achieve high performance and strengthen system's robustness, we proposed a fuzzy ADRC controller by designing an extended state observer (ESO) and fuzzy nonlinear feedback control law. The proposed attitude control scheme is shown in Figure 1.

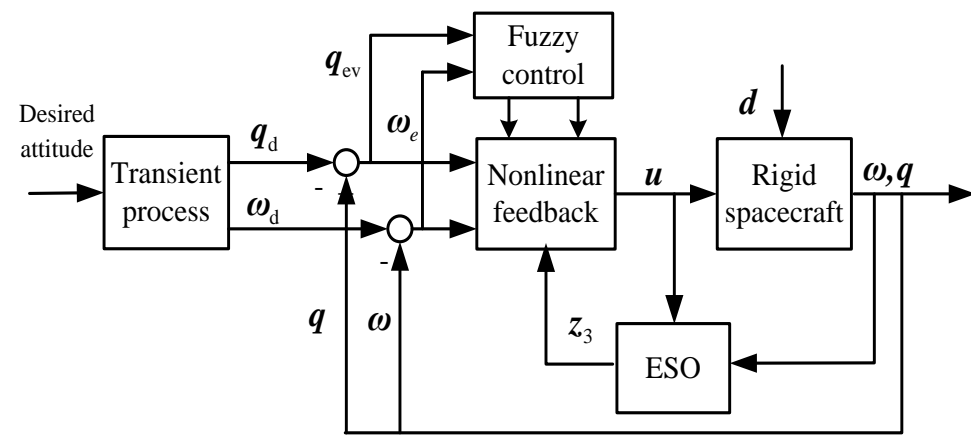

Figure. 1 Attitude Control Scheme of Spacecraft

\subsection{Design of the ESO}

The critical point of ADRC is treating total disturbance, including the external disturbances and parametric uncertainties, as an extended state variable. By designing the ESO for the spacecraft attitude control system, we estimate the total disturbance online by extending a state variable.

First step, the attitude dynamic equation (3) and (4) can be transformed into a second-order nonlinear system as follows:

$$
\begin{aligned}
& \dot{\boldsymbol{q}}=\frac{1}{2} \boldsymbol{q} \otimes \boldsymbol{\omega} \\
& \dot{\boldsymbol{\omega}}=-\boldsymbol{J}_{0}^{-1} \boldsymbol{\omega}^{\times} \boldsymbol{J}_{0} \boldsymbol{\omega}+\boldsymbol{J}_{0}^{-1} \boldsymbol{u}+\boldsymbol{f}
\end{aligned}
$$

where $\boldsymbol{q} \in R^{4 \times 1}, \boldsymbol{\omega} \in R^{3 \times 1}$, and $\boldsymbol{f}=-\boldsymbol{J}_{0}^{-1} \boldsymbol{d}-\boldsymbol{J}_{0}^{-1} \boldsymbol{\omega}^{\times} \boldsymbol{J}_{\Delta} \boldsymbol{\omega}-\boldsymbol{J}_{0}^{-1} \boldsymbol{J}_{\Delta} \dot{\boldsymbol{\omega}}, \boldsymbol{f}$ can be considered as the total disturbance .

Then, for system (8), we design a nonlinear third-order extended state observer as follows, so that the "total disturbance" can be estimated.

$$
\begin{aligned}
& \dot{z}_{1}=\frac{1}{2} \boldsymbol{q} \otimes\left[\begin{array}{ll}
\boldsymbol{z}_{2}^{\mathrm{T}} & 0
\end{array}\right]^{\mathrm{T}}-\beta_{1} \boldsymbol{e} \\
& \dot{z}_{2}=\boldsymbol{z}_{3}+\boldsymbol{J}_{0}^{-1} \boldsymbol{u}-\beta_{2} \operatorname{fal}\left(\boldsymbol{e}_{\mathrm{v}}, \alpha, \delta\right) \\
& \dot{\boldsymbol{z}}_{3}=-\beta_{3} \operatorname{fal}\left(\boldsymbol{e}_{\mathrm{v}}, \alpha, \delta\right)
\end{aligned}
$$


where $\boldsymbol{e}=z_{1}-\boldsymbol{q}, \boldsymbol{e}=\left[\begin{array}{llll}e_{1} & e_{2} & e_{3} & e_{4}\end{array}\right]^{\mathrm{T}}, \boldsymbol{e}_{\mathrm{v}}=\left[\begin{array}{lll}e_{1} & e_{2} & e_{3}\end{array}\right]^{\mathrm{T}}, z_{1} \in R^{4 \times 1}, z_{2} \in R^{3 \times 1}, z_{3} \in R^{3 \times 1}$, $\beta_{i} \in R^{+}, i=1,2,3, \quad f a l(e, \alpha, \delta): R^{3} \rightarrow R, \quad f a l(e, \alpha, \delta)=\left\{\begin{array}{cl}e \delta^{\alpha-1}, & |e| \leq \delta \\ |e|^{\alpha} \operatorname{sign}(e), & |e|>\delta\end{array}, \quad e_{\mathrm{i}} \in R\right.$, $\alpha \in R^{+} \quad, \quad \delta \in R^{+} \quad$. If $\boldsymbol{e}=\left[e_{1}, e_{2}, \cdots e_{n}\right]^{\mathrm{T}} \quad$ is a vector, $f a l(\boldsymbol{e}, \alpha, \delta)=\left[f a l\left(e_{1}, \alpha, \delta\right), f a l\left(e_{2}, \alpha, \delta\right) \cdots f a l\left(e_{n}, \alpha, \delta\right)\right]$. According to the trial and error method, there was a control strategy for the gain in $f a l(\square)$ : the gain is set at a small value when the error is large, and vice versa. Here, the parameters $\alpha, \delta, \beta_{1}, \beta_{2}, \beta_{3}$ of the extended state observer are usually set as constants. Where $\alpha$ is a positive coefficient, $\delta=h$ and $h$ is sample period of the controller; It was indicated in ref. [12] that $\beta_{1}, \beta_{2}$ and $\beta_{3}$ can be also determined by the sample period, and in general $\beta_{1}=1 / h, \beta_{2}=1 / 3 h^{2}$ and $\beta_{3}=1 / 8 h^{3}$ are recommended for applications. The inputs of the ESO are $\boldsymbol{e}, \boldsymbol{q}, \boldsymbol{\omega}$ and $\boldsymbol{u}$, and the outputs of the ESO $\boldsymbol{z}_{i}, i=1,2,3$ are the estimations of the attitude quaternion $q$, angular velocity $\omega$ and total disturbance $f$, respectively. According to ref. [13,14], $z_{1} \approx \boldsymbol{q}, \boldsymbol{z}_{2} \approx \boldsymbol{\omega}, \boldsymbol{z}_{3} \approx \boldsymbol{f}$ can be realized by choosing proper parameters of ESO appropriately.

\subsection{Fuzzy Nonlinear Feedback Control Law}

The parameters of nonlinear feedback control law in convention ADRC are usually defined as constants which are set offline. However, if the ADRC with constant parameters was applied in the spacecraft attitude maneuver control, it would heavily affect the dynamic performance of the system, because it cannot adapt to the change of control system states when attitude error $\boldsymbol{q}_{e v}$ and angular velocity error $\boldsymbol{\omega}_{e}$ vary greatly during attitude maneuver. However, setting the magnitude of each parameter manually is time-consuming and not conducive to the actual operation. Aiming at enhancing the adaptive capacity and improving dynamic performance of the system, we introduce fuzzy logic control, which has reasoning capacity and is independent of detailed mathematical formulations, to adjust the parameters in feedback control law online. Based on feedback information $\boldsymbol{q}_{\mathrm{ev}}, \boldsymbol{\omega}_{\mathrm{e}}$ and the estimation information $z_{3}$ from the ESO, we design a fuzzy nonlinear feedback and compensation control law as follows:

$$
\boldsymbol{u}=\boldsymbol{J}_{0}\left(-\boldsymbol{k}_{1} f a l\left(\boldsymbol{q}_{\mathrm{ev}}, \alpha_{1}, h\right)-\boldsymbol{k}_{2} f a l\left(\boldsymbol{\omega}_{e}, \alpha_{2}, h\right)-\boldsymbol{z}_{3}\right)
$$

where $\alpha_{1}, \alpha_{2}$ are positive constants, $\alpha_{1}, \alpha_{2}$ satisfy $\alpha_{1}<\alpha_{2}<1, h$ is sample period. $\boldsymbol{k}_{\boldsymbol{1}}=\operatorname{diag}\left(k_{1 i}\right), \quad \boldsymbol{k}_{2}=\operatorname{diag}\left(k_{2 i}\right), i=1,2,3 . k_{1 i}$ and $k_{2 i}$ are the tunable parameters adjusted by fuzzy logic controllers. Here, $z_{3}$ is a compensation term for the total disturbance, and $-\boldsymbol{k}_{1} f a l\left(\boldsymbol{q}_{\mathrm{ev}}, \alpha_{1}, h\right)-\boldsymbol{k}_{2} f a l\left(\boldsymbol{\omega}_{e}, \alpha_{2}, h\right)$ is a nonlinear feedback control term to track the planned attitude maneuver path rapidly.

Fuzzy self-adjusting parameters $\Delta k_{1 i}$ and $\Delta k_{2 i}$ are introduced in the nonlinear feedback law (10), and the parameters $k_{1 i}$ and $k_{2 i}$ can be written as:

$$
\left\{\begin{array}{l}
k_{1 i}=k_{1}+\Delta k_{1 i}, \quad \Delta k_{1 i}=f_{1}\left(q_{e i}, \omega_{e i}\right) \\
k_{2 i}=k_{2}+\Delta k_{2 i}, \quad \Delta k_{2 i}=f_{2}\left(q_{e i}, \omega_{e i}\right)
\end{array}, i=1,2,3\right.
$$

where $k_{1}$ and $k_{2}$ are base values of $k_{1 i}$ and $k_{2 i}$ respectively, which are set appropriately. $f_{1}(\square): R^{2} \rightarrow R$ and $f_{2}(\square): R^{2} \rightarrow R$ are fuzzy functions. 
In the fuzzy functions, the quaternion attitude angular error $q_{e i}$ and angular velocity error $\omega_{e i}$ are input variables and both of them range from -1 to 1 . Meanwhile, the output variables of fuzzy functions are $\Delta k_{1 i}$ and $\Delta k_{2 i}$ which range from -3 to 3. Each of the above variables is divided into seven language sets defined as: $\{\mathrm{NB}$ (negative big), NM(negative medium), NS(negative small), ZE(zero), PS(positive small), $\mathrm{PM}$ (positive medium), $\mathrm{PB}$ (positive big)\}. The input variables are characterized by the Gaussian membership function and the output variables are characterized by the triangular membership function. The fuzzy logic controller use Mamdani type; and bisector method is used for Defuzzification. Through summarizing the practice engineering experience and expert knowledge [15], fuzzy logic rules can be worked out. The fuzzy rules of $\Delta k_{1 i}$ and $\Delta k_{2 i}$ are established and given in table 1 and table 2 respectively.

Table 1. Fuzzy Rules Of $\Delta k_{1 i}$

\begin{tabular}{|c|c|c|c|c|c|c|c|}
\hline \multirow{3}{*}{$\Delta k_{1 i}$} & \multicolumn{7}{|c|}{$\omega_{e i}$} \\
\hline & $\mathrm{N}$ & $\mathrm{N}$ & $\mathrm{N}$ & $7 \mathrm{~F}$ & $\mathrm{P}$ & $\mathrm{P}$ & $\mathrm{PB}$ \\
\hline & B & $\mathrm{M}$ & $\mathrm{S}$ & & $\mathrm{S}$ & $\mathrm{M}$ & \\
\hline $\mathrm{N}$ & $\mathrm{P}$ & $\mathrm{P}$ & $\mathrm{P}$ & DM & $\mathrm{P}$ & $\mathrm{Z}$ & ZE \\
\hline B & B & B & M & FIV & $\mathrm{S}$ & $\mathrm{E}$ & \\
\hline $\mathrm{N}$ & $\mathrm{P}$ & $\mathrm{P}$ & $\mathrm{P}$ & $D C$ & $\mathrm{P}$ & $Z$ & $\overline{Z E}$ \\
\hline M & B & B & M & PS & $\mathrm{S}$ & $\mathrm{E}$ & \\
\hline $\mathrm{N}$ & $\mathrm{P}$ & $\mathrm{P}$ & $\mathrm{P}$ & PM & $Z$ & $\mathrm{~N}$ & NS \\
\hline$S$ & $\mathrm{M}$ & M & M & PM & $\mathrm{E}$ & S & \\
\hline Z Z & $\mathrm{P}$ & $\mathrm{P}$ & $\mathrm{P}$ & & $\mathrm{N}$ & $\mathrm{N}$ & $\mathrm{N}$ \\
\hline $\begin{array}{ll}q, & \mathrm{E}\end{array}$ & $M$ & M & $\mathrm{S}$ & $\mathrm{ZE}$ & $\mathrm{S}$ & $\mathrm{M}$ & M \\
\hline $\mathrm{P}$ & $\mathrm{P}$ & $P$ & $\mathrm{Z}$ & & $\mathrm{N}$ & $\mathrm{N}$ & $\mathrm{N}$ \\
\hline S & $\mathrm{S}$ & $\mathrm{S}$ & $\mathrm{E}$ & NS & $\mathrm{S}$ & $\mathrm{M}$ & M \\
\hline $\mathrm{P}$ & $\mathrm{P}$ & Z & $\mathrm{N}$ & $\mathrm{N}$ & $\mathrm{N}$ & $\mathrm{N}$ & $\mathrm{NB}$ \\
\hline M & $\mathrm{S}$ & $\mathrm{E}$ & $\mathrm{S}$ & $\mathrm{M}$ & M & M & \\
\hline $\mathrm{P}$ & Z & Z & $\mathrm{N}$ & $\mathrm{N}$ & $\mathrm{N}$ & $\mathrm{N}$ & $\mathrm{NB}$ \\
\hline B & $\mathrm{E}$ & $\mathrm{E}$ & M & M & $\mathrm{M}$ & B & \\
\hline
\end{tabular}

Table 2. Fuzzy Rules Of $\Delta k_{2 i}$

\begin{tabular}{|c|c|c|c|c|c|c|c|}
\hline \multirow[b]{2}{*}{$\Delta k_{2 i}$} & \multicolumn{7}{|c|}{$\omega_{e i}$} \\
\hline & $\mathrm{B}^{\mathrm{N}}$ & $\mathrm{M}^{\mathrm{N}}$ & $\begin{array}{c}\mathrm{S}^{\mathrm{N}} \\
\text {. }\end{array}$ & ZE & $\begin{array}{l}{ }_{S}{ }^{P} \\
\text {. }\end{array}$ & $\begin{array}{c}\mathrm{P} \\
\mathrm{M}^{-}\end{array}$ & PB \\
\hline $\mathrm{N}$ & $\mathrm{P}$ & $\mathrm{N}$ & $\mathrm{N}$ & NDP & $\mathrm{N}$ & $\mathrm{N}$ & PS \\
\hline B & $\mathrm{S}$ & $\mathrm{S}$ & B & NDD & B & $\mathrm{M}$ & \\
\hline $\mathrm{N}$ & $\mathrm{P}$ & $\mathrm{N}$ & $\mathrm{N}$ & NID & $\mathrm{N}$ & $\mathrm{N}$ & PS \\
\hline $\mathrm{M}$ & $\mathrm{S}$ & $\mathrm{S}$ & B & NDB & B & $\mathrm{M}$ & \\
\hline $\mathrm{N}$ & $Z$ & $\mathrm{~N}$ & $\mathrm{~N}$ & $\mathrm{~N}$ & $\mathrm{~N}$ & $\mathrm{~N}$ & $\mathrm{ZE}$ \\
\hline $\mathrm{S}$ & $\mathrm{E}$ & $\mathrm{S}$ & $\mathrm{M}$ & $\mathrm{M}$ & $\mathrm{S}$ & $S$ & \\
\hline & $\mathrm{Z}$ & $\mathrm{N}$ & $\mathrm{N}$ & NS & $\mathrm{N}$ & $\mathrm{N}$ & $\mathrm{ZE}$ \\
\hline $\begin{array}{ll}11 & \mathrm{E}\end{array}$ & $\mathrm{E}$ & $\mathrm{S}$ & S & NS & $\mathrm{S}$ & $S$ & \\
\hline $\mathrm{P}$ & Z & Z & Z & $7 \mathrm{~F}$ & Z & $\mathrm{Z}$ & $\mathrm{ZE}$ \\
\hline $\mathrm{S}$ & $\mathrm{E}$ & $E$ & $\mathrm{E}$ & $\angle E$ & $E$ & $E$ & \\
\hline $\mathrm{P}$ & $\mathrm{P}$ & $\mathrm{N}$ & $\mathrm{P}$ & $\mathrm{DS}$ & $\mathrm{P}$ & $\mathrm{P}$ & PB \\
\hline $\mathrm{M}$ & B & $\mathrm{S}$ & $S$ & PS & $\mathrm{S}$ & $S$ & \\
\hline $\bar{P}$ & $\mathrm{P}$ & $\mathrm{P}$ & $\mathrm{P}$ & PM & $\mathrm{P}$ & $\mathrm{P}$ & PB \\
\hline B & B & $\mathrm{M}$ & $\mathrm{M}$ & IVI & $\mathrm{S}$ & $\mathrm{S}$ & \\
\hline
\end{tabular}




\section{Simulation Results and Analysis}

In this section, a case study of a rigid spacecraft is employed to illustrate the effectiveness and robustness of the proposed fuzzy ADRC scheme, in which the spacecraft is commanded to perform a Rest-to-Rest three-axis attitude maneuver. In this case, the parameters of the spacecraft are taken from [16] and the external disturbance, including constant disturbance and periodic disturbance with three kinds of frequency, is taken into account as follows:

$$
\boldsymbol{d}=\left[\begin{array}{c}
-3+4 \cos (0.2 \pi t)-\cos (0.4 \pi t)+2 \omega_{1} \sin (0.11 t) \\
4+3 \sin (0.2 \pi t)-2 \cos (0.4 \pi t)+2 \omega_{2} \sin (0.11 t) \\
-3+4 \sin (0.2 \pi t)-3 \sin (0.4 \pi t)-2 \omega_{3} \sin (0.11 t)
\end{array}\right] \times 10^{-4} \text { N.m. }
$$

And the parametric uncertainty in the inertia matrix of spacecraft model also is considered as follows:

$$
\boldsymbol{J}=\boldsymbol{J}_{0}+\boldsymbol{J}_{\Delta}, \boldsymbol{J}_{0}=\left[\begin{array}{ccc}
350 & 3 & 4 \\
3 & 270 & 10 \\
4 & 10 & 190
\end{array}\right]\left(\mathrm{kg}\left[\mathrm{m}^{2}\right), \boldsymbol{J}_{\Delta}=0.2 \boldsymbol{J}_{0}\right.
$$

The initial attitude in Euler angles is $\left[\begin{array}{lll}10^{\circ} & 15^{\circ} & -10^{\circ}\end{array}\right]^{\mathrm{T}}$ and the desired attitude is $\left[\begin{array}{lll}0^{\circ} & 0^{\circ} & 0^{\circ}\end{array}\right]^{\mathrm{T}}$. The initial and desired attitude angular velocity vector is $[0 \% \mathrm{~s} 0 \% \mathrm{~s} \quad 0 \%]^{\mathrm{T}}$, and the span of simulation time is from 0s to 100s. The maximum control torque is supposed 10 (N.m).

The parameter of expected transient process is $a=0.2^{\circ} / s^{2}$. The parameters of the extended state observer

are $\delta=h=0.02, \beta_{1}=1 / h=50, \beta_{2}=1 / 3 h^{2}=833, \beta_{3}=1 / 8 h^{3}=15625, \alpha=0.5$. The parameters in the fuzzy nonlinear feedback control law are $\alpha_{1}=0.5, \alpha_{2}=0.6$, and the base values of $k_{1 i}$ and $k_{2 i}$ are chosen as $k_{1}=5$ and $k_{2}=3$. The simulation results have been shown in Figure 2 - Figure. 4.

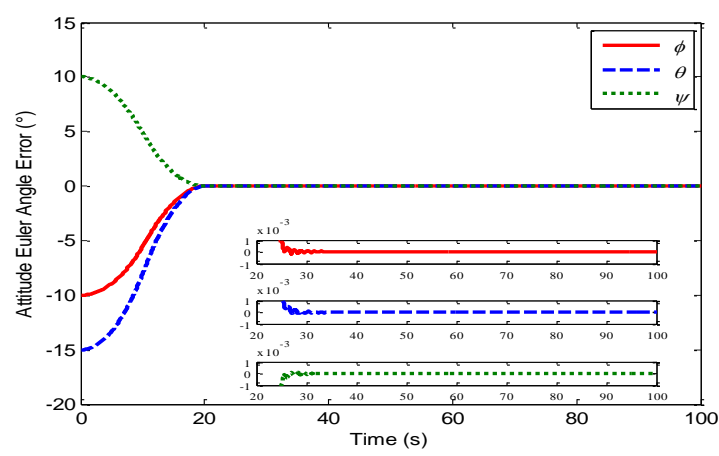

Figure. 2. Response of Euler Angle Error 


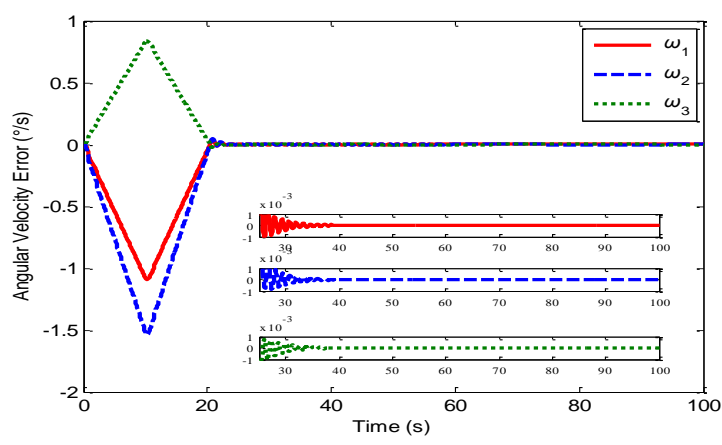

Figure 3. Response of Angular Velocity Error


Figure 4. Profile of Control Torque

It can be seen from Figure. 2 3 that it takes about 20 seconds for the attitude maneuver with the proposed control scheme. And the overshoot is effectively reduced during the initial period of the attitude maneuver. Through the zoomed version of errors, it is known that the attitude angular error and angular velocity error are kept within a range of less than $1 \times 10^{-3}\left({ }^{\circ}\right)$ and $1 \times 10^{-3}(\%)$ after about 25 seconds, and both of them converge to zero. Thus, it can be concluded that the proposed controller is workable for rapid attitude maneuver. Moreover, the attitude control system is of high pointing precision with rather good stability after completing the maneuver. Figure. 4 shows that control torques are smooth and the maximum control torque is less than 2(N.m).

In addition, to verify the robustness of the proposed controller, it is supposed that the magnitude of external disturbances increase fivefold in the following simulation. In Table 3, the comparison of stable time $t_{\mathrm{s}}$, pointing accuracy $P_{\mathrm{m}}$ and stability $P_{\mathrm{s}}$ with these two different external disturbances are given and the definition of the performance indicators are defined herein as follows: (1) $t_{\mathrm{s}}$ is the time required for both of the single-axis attitude angular error and the attitude angular velocity error converge into the range of $5 \times 10^{-4}\left({ }^{\circ}\right)$ and $5 \times 10^{-4}(\%)$. (2) $P_{\mathrm{m}}$ is the steady state pointing accuracy which is defined as the square error of single-axis attitude angle from 50s to $100 \mathrm{~s}$. (3) $P_{\mathrm{s}}$ is the standard deviation of single-axis attitude angular velocity from $50 \mathrm{~s}$ to $100 \mathrm{~s}$, which is used to evaluate the stability of the control system. It can be observed from Table 3 that the performance indicators vary only a little when the external disturbances increase fivefold, which implies the proposed controller has high robustness to external disturbances. 
Table 3. Performance Indicators on Different External Disturbance

\begin{tabular}{ccccccccccccc}
\hline External & \multicolumn{3}{c}{$t_{\mathrm{s}}(\mathrm{s})$} & \multicolumn{3}{c}{$P_{\mathrm{m}} \times 10^{-5}\left({ }^{\circ}\right)$} & \multicolumn{3}{c}{$P_{\mathrm{s}} \times 10^{-6}\left({ }^{\circ} / \mathrm{s}\right)$} \\
Disturbance & \multicolumn{2}{c}{$\mathrm{x}$} & $\mathrm{y}$ & $\mathrm{z}$ & $\mathrm{x}$ & $\mathrm{y}$ & $\mathrm{z}$ & $\mathrm{x}$ & $\mathrm{y}$ & $\mathrm{z}$ \\
\hline $\boldsymbol{d}$ & 24 & 24 & 22 & 0.72 & 0.55 & 1.94 & 0.53 & 0.48 & 1.08 \\
$5 \boldsymbol{d}$ & 24 & 24 & 22 & & 0.21 & 1.76 & 3.82 & 1.10 & 0.86 & 2.13 \\
\hline
\end{tabular}

In practice, the inertia matrix of orbiting spacecraft varies, which may heavily affect the performance of the attitude control system. Table 4 makes a comparison of performance indicators while the inertia matrix varies. It can be seen that the performance indexes vary only a little, which implies that the proposed controller has a good robustness to the parameter uncertainties in inertia matrix.

Table 4. Performance Indicators on Different Inertia

\begin{tabular}{lcccccccccccc}
\hline Inertia & \multicolumn{1}{c}{$t_{\mathrm{s}}(\mathrm{s})$} & \multicolumn{8}{c}{$P_{\mathrm{m}} \times 10^{-5}\left({ }^{\circ}\right)$} & \multicolumn{6}{c}{$P_{\mathrm{s}} \times 10^{-6}\left({ }^{\circ} / \mathrm{s}\right)$} \\
Matrix & $\mathrm{x}$ & $\mathrm{y}$ & $\mathrm{z}$ & $\mathrm{x}$ & $\mathrm{y}$ & $\mathrm{z}$ & $\mathrm{x}$ & $\mathrm{y}$ & $\mathrm{z}$ & \\
\hline & $1.2 \boldsymbol{J}$ & \multicolumn{2}{c}{24} & 24 & 22 & 0.65 & 0.50 & 1.76 & 2.08 & 1.35 & 1.61 \\
$\boldsymbol{J}$ & 24 & 24 & 22 & 0.72 & 0.55 & 1.94 & 0.53 & 0.48 & 1.08 & \\
$0.8 \boldsymbol{J}$ & 24 & 24 & 22 & 0.90 & 0.69 & 2.42 & 0.42 & 0.27 & 1.29 & \\
\hline
\end{tabular}

\section{Conclusion}

In this paper, a novel fuzzy ADRC control scheme is proposed for attitude maneuver of the spacecraft with unknown disturbances and parametric uncertainties. The proposed control strategy has following advantages: By designing transient process of the maneuver path, the contradictory between overshoot and rapidity can be eased. The controller does not require any prior information about the external disturbances and parametric uncertainties, and it can estimate and compensate for them effectively. With the advantages of fuzzy logic control, the parameters in the feedback control laws can be self-adjusting according to system state variation and thus the dynamic performance of control system is improved. Finally, simulation results demonstrated the effectiveness and robustness of the proposed control scheme.

\section{Acknowledgements}

This work was supported by the National Natural Science Foundation of China under grant 61473152, the China Scholarship Council (CSC), the Innovation Project for College Graduates of Jiangsu Province No.CXLX13_190 and the Educational Innovation Project for Graduate Students of Nanjing University of Science and Technology.

\section{References}

[1] S. C. Greenland and W. J. Crowther, "Development of an attitude guidance and control system for the Thunderstar suborbital spacecraft," Journal of Aerospace Engineering, vol. 221, no. 2, (2007), pp. 259-272.

[2] Z. Y. Chen and J Huang. "Attitude tracking and disturbance rejection of rigid spacecraft by adaptive control," IEEE Transactions on Automatic Control, vol. 54, no. 3, (2009), pp. 600-605.

[3] K. S. Kim and Y. Kim. "Robust backstepping control for slew maneuver using nonlinear tracking function." IEEE Transactions on Control Systems Technology, vol. 11, no. 6 (2003), pp. 822-829.

[4] Y. Xia, Z. Zhu, M. Fu and S. Wang. "Attitude tracking of rigid spacecraft with bounded disturbances," IEEE Transactions on Industrial Electronics, vol. 58, no. 2, (2011), pp. 647-659.

[5] Y Park. "Inverse optimal and robust nonlinear attitude control of rigid spacecraft," Aerospace Science and Technology, vol. 28, no. 1, (2013), pp: 257-265. 
[6] Y Guo, C. F. Zhou, Z. Yu, and Q. W. Chen. "Study on attitude control for move-to-rest maneuver of flexible spacecraft," International Journal of Modelling, Identification and Control, 2013. Vol. 19, no. 1, (2013), pp. 23-31.

[7] J. Q. Han, "From PID to active disturbance rejection control," IEEE Transactions on Industrial Electronics, vol. 56, no. 3, (2009), pp. 900-906.

[8] Z. Gao, S. Hu and F. Jiang. "A novel motion control design approach based on active disturbance rejection." In Proceedings of the 40th IEEE Conference on Decision and Control, vol. 5, Orlando, USA, (2001), pp. 4877-4882.

[9] A. F. Lai, Y. Guo, and L. J. Zheng, "Spacecraft attitude control with high precision and high stability based on active disturbance rejection technique," Control Theory and Applications, vol. 29, no. 3, (2012), pp.401-407.

[10] C. X. Zhong, A. F. Lai, Y Guo and Q. W. Chen. "On Attitude Maneuver Control of Flexible Spacecraft without Angular Velocity Sensors." In Proceedings of the 2013IEEE/SICE International Symposium on System Integration, Kobe, Japan, (2013), pp. 318-323.

[11] C. Y. Zhu, D. Yang and K Zhai. "Active disturbance rejection attitude controller for large flexible multi-body satellite without gyroscopes." Aerospace Control, vol. 22, no. 1, (2005), pp. 43-47.

[12] J. Q. Han. Active disturbance rejection control technique. National Defence Industry Press, Beijing (2008).

[13] J. Q. Han, “The 'extended state observer' of a class of uncertain systems," Control and Decision, vol. 10, no. 1, (1995), pp.85-88.

[14] B. Z. Guo and Z. L. Zhao. "On the convergence of an extended state observer for nonlinear systems with uncertainty." Systems \& Control Letters, vol. 60, no. 6, (2011), pp. 420-430.

[15] S. Huang, J. Kuang, Q. Huang, J. Gao, and T. Liu. "IPMSM sensor less control based on fuzzy active-disturbance rejection controller for electric vehicle" In Power Engineering, Energy and Electrical Drives (POWERENG), 2011 International Conference on. IEEE, Malaga, Spain, (2011), pp. 1-6.

[16] S. D. Gennaro, "Output stabilization of flexible spacecraft with active vibration suppression," IEEE Transactions on Aerospace and Electronic systems, vol. 39, no. 3, (2003), pp. 747-759.

\section{Authors}

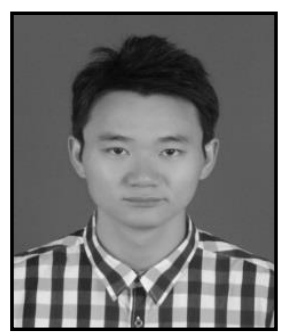

Chenxing Zhong received his BSc in 2011 in Electrical Engineering and Automation from Nanjing University of Science and Technology, Nanjing, China. He is currently a $\mathrm{PhD}$ candidate in Control Theory and Control Engineering at Nanjing University of Science and Technology. His research interests include spacecraft attitude control, nonlinear control and vibration control.



Yu Guo received her BSc (1984) and MSc (1987) in Automation, both from Huazhong University of Science and Technology, Wuhan, China, and her PhD in Control Science and Engineering from Nanjing University of Science and Technology. In 1987, she joined the faculty of the School of Automation, Nanjing University of Science and Technology, and is currently a Professor of Automatic Control there. Her main research interests are adaptive control, robust control, nonlinear control and multiobjective optimization for complicated systems.

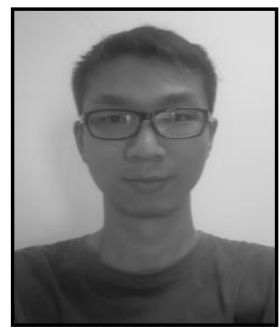

Lu Wang received his BSc in 2012 in Electrical Engineering and Automation from Nanjing University of Science and Technology, Nanjing, China. He is currently a $\mathrm{PhD}$ candidate in Control Theory and Control Engineering at Nanjing University of Science and Technology. His research interests include design of steering law of single-gimbaled control moment gyroscopes (SGCMGs), spacecraft attitude control considering actuator and non-linear control. 